\title{
EL LIDERAZGO EN LA UNIVERSIDAD: EL PAPEL QUE TIENEN ELLAS
}

\section{LEADERSHIP IN UNIVERSITY: THE ROLE THAT WOMEN HAVE}

\author{
Marina TOMÀS-FOLCH \\ Universitat Autònoma de Barcelona \\ marina.tomas@uab.cat \\ orcid.org/0000-0001-5445-4823
}

\section{Resumen}

En este artículo presentamos tres factores que determinan en gran parte la igualdad de oportunidades en las universidades con respecto al género: la cultura organizativa en la Universidad, la participación en la gestión universitaria y el ejercicio del liderazgo. Se presentan tres estudios mixtos (cuantitativos y cualitativos) cuyo objetivo es obtener la percepción que tiene el profesorado universitario de la cultura institucional con respecto al género, al liderazgo y a la participación en la toma de decisiones. Los resultados muestran que persiste una percepción sobre modelos estereotipados del estilo de liderazgo de las personas que ejercen la dirección departamental que se identifican con la construcción social de lo masculino, que existen formas de participación en las reuniones de los órganos de gobierno diferenciados según el género de quien participa y de quien lidera, que se encuentran ciertos rasgos en las motivaciones y formas de ejercer los cargos de dirección en la Universidad en función del género y que el liderazgo de la investigación está mayoritariamente en manos del género masculino.

Palabras clave: liderazgo, Universidad, mujeres, participación, toma de decisiones.

\begin{abstract}
In this article we present three factors that determine in great part the equality of opportunities in universities with respect to the gender: the organizational culture in the university, the participation in the university management and the exercise of leadership. Quantitative and qualitative studies have been carried out in order to obtain professors' perception of the institutional culture regarding gender, leadership and
\end{abstract}


participation in decision making. The results show that there's a persistant perception about stereotyped models of the leadership style of the departmental leaders identified with the social construction of the masculine, that there are forms of participation in the meetings of the governing bodies differentiated by the gender of the professors who participate and by the professor who lead, that certain features are found in the motivations and ways of exercising management positions in the university according to gender and that the leadership of the research is mostly in the hands of the male gender.

Keywords: leadership, university, women, participation, decision making. 


\section{INTRODUCCIÓN}

En este artículo presentamos, de una manera transversal, los resultados de tres investigaciones realizadas por equipos de investigación dirigidos por la autora con el correspondiente estado de la cuestión respecto a los tópicos de: liderazgo en la Universidad tanto en la gestión académica como en la investigación, la participación en la toma de decisiones y la cultura organizativa.

\subsection{La cultura universitaria: una rémora para la igualdad de oportunidades de género}

Desde la mirada de la cultura organizacional universitaria, es lógico que nos interesemos por su relación con el género. El género es una construcción cultural y, como tal, debería aparecer como elemento indispensable en cualquier análisis de la cultura organizativa. En la Universidad, como en cualquier otra organización, se construyen y reproducen significados culturales con un contenido de género. Algunos análisis sugieren que las culturas organizativas suelen privilegiar significados masculinos que son adoptados como referentes de lo que es «normal», «natural» o simplemente valioso en las organizaciones.

Respecto a las razones extrínsecas que explican la desigual e infrarrepresentada presencia de las mujeres en los cargos directivos de la Universidad observamos la existencia de una cultura masculinizada que frena la iniciativa de la mujer para acceder a cargos de gestión. Las mujeres manifiestan tener que demostrar ser muy buenas en el ejercicio de sus roles para ser reconocidas y aceptadas en sus cargos de gestión. Estos hallazgos coinciden con los expuestos por Carli y Eagly y Johannsen-Schmidt para explicar la (in)visibilidad de las mujeres en puestos de poder así como con los de Kram y Humpton quienes consideran que el predominio de una cultura masculinizada constituye una dificultad para que las mujeres accedan a posiciones de poder en las universidades (Sánchez-Moreno, Tomàs-Folch y Lavié).

Sin embargo, salvo contadas excepciones, los análisis de las culturas organizativas son notorios por su falta de atención a las cuestiones relacionadas con el género. Esto, por otra parte, tampoco debería extrañarnos: la teoría 
organizativa ha estado tradicionalmente de espaldas a las cuestiones de género, y sólo en los últimos años ha comenzado a desarrollar una mayor sensibilidad hacia tópicos relacionados con el mismo. Los análisis de género se han preocupado tradicionalmente por cuestiones de representatividad numérica o por las formas distintivas de hacer de las mujeres en puestos de gestión. Sin desmerecer estos planteamientos, el estudio de la Universidad desde una perspectiva de género debe estar guiado también por un interés por desvelar el sustrato de género que informa a los significados culturales. En definitiva, sobre la cuestión del análisis de la cultura organizativa en la Universidad desde una óptica de género podemos concluir lo siguiente:

- No disponemos de abundantes estudios sobre cultura organizativa y género, si bien es previsible que la incorporación de una perspectiva de género en el análisis de la cultura organizativa haga visible la manera en que las culturas y los elementos simbólicos de una organización pueden marginalizar a las mujeres.

- La dificultad de identificar y definir las barreras culturales relacionadas con el género, como elementos simbólicos, no debe ignorar su análisis y estudio en profundidad.

La investigación en torno al techo de cristal ha puesto de manifiesto las múltiples dificultades con las que se encuentran muchas mujeres en su progresión en la escala organizativa. En el contexto universitario las cifras son concluyentes, el género persiste como criterio diferenciador de poder. Así pues será preciso «repensar» nuestra propia cultura organizacional, para conocer los roles inter géneros y la aceptación de cada uno de ellos, fortaleciendo la institución desde el enriquecimiento por:

- Los preocupados por identificar las barreras que encuentran las mujeres en sus intentos por avanzar profesionalmente en el marco de la dirección.

- Aquellos interesados en documentar las estrategias puestas en juego por las mujeres que logran cierto éxito en el contexto empresarial.

- Los que procuran definir un «estilo femenino» en el ejercicio del liderazgo que aporta la diversidad de ideas, valores, costumbres, hábitos y formas de proceder (Caceres, Sachicola e Hinojo). 


\subsection{El liderazgo y la dualidad «igualdad versus diferencia entre géneros»}

En el campo de las organizaciones empresariales, a diferencia de lo que ha sucedido en el de las educativas, muchas investigaciones se han preocupado de analizar la incidencia de la variable género. Su enfoque - fiel a la perspectiva tecnocrática que acostumbran a adoptar este tipo de estudios- ha sido predominantemente descriptivo de las diferencias en el comportamiento, el trato o el desarrollo de los hombres y de las mujeres en estas organizaciones.

Desde el liderazgo como también desde otras facetas profesionales se considera la dualidad «igualdad versus diferencia» entre géneros. En un extremo encontramos lo que podríamos llamar un enfoque centrado en reclamar la igualdad de hombres y mujeres en el ejercicio del liderazgo y en el otro, los enfoques orientados a reclamar la diferencia. De acuerdo con el primer enfoque, hombres y mujeres son esencialmente iguales, por lo que el problema puede definirse como de acceso a estructuras y procesos que se consideran neutrales. La desigualdad de las mujeres se entiende, pues, como resultado de restricciones de orden social e institucional, que se pueden erradicar desmantelando estas barreras estructurales a la igualdad. La lógica subyacente a este discurso es que, al eliminar las barreras estructurales y procedimentales, una buena parte de mujeres tendrá acceso, por su propio mérito, al poder formal. Desde este enfoque, por tanto, hermanado con la teoría política liberal y el feminismo liberal, se ha reclamado el acceso a puestos de responsabilidad basado en el mérito individual. Las estrategias de igualdad de género promovidas desde esta perspectiva se centran fundamentalmente en cambiar a las mujeres de manera individual, no a las culturas donde trabajan, para que se parezcan más a los hombres: formación para adquirir las destrezas propias de un campo del que, hasta el momento, habían sido ajenas. Para asegurar la igualdad de oportunidades, las mujeres deben ser formadas para cubrir las carencias propias de su socialización diferencial y llegar, de este modo, a un nivel y desempeño comparable al de sus colegas varones. Hay que desarrollar las destrezas y estilos que se consideran necesarios para el éxito. Desde esta perspectiva, el feminismo liberal ha perseguido la eliminación de los estereotipos asociadas al género.

De acuerdo con esta perspectiva, las mujeres con éxito en puestos de liderazgo son las que logran una integración -en lugar de polarización- de rasgos tanto femeninos como masculinos (Park). Se resuelven de esta manera las percepciones negativas que suelen atribuirse al liderazgo femenino, una de las principales razones argumentadas para justificar la escasa representación de las mujeres en los puestos de gestión educativa. En el extremo opuesto, a diferencia de lo que ocurre con los planteamientos que buscan mejorar 
las deficiencias de la mujer para el liderazgo, se ensalza la diferencia como constitutiva del género, y se enfatiza el valor positivo de las cualidades tradicionalmente asociadas a las mujeres. Partiendo de esta diferenciación clásica de los roles femeninos y masculinos, se asume que hay ciertas cualidades que se identifican con un estilo de liderazgo «femenino»o «masculino». Aunque cualquiera de estos estilos puede ser adoptado tanto por hombres como por mujeres, la asunción básica y las expectativas que de ella se derivan es que los hombres desarrollan un estilo predominantemente masculino, mientras que las mujeres uno fundamentalmente femenino. De acuerdo con esta asunción, los hombres en puestos de responsabilidad tienden a desarrollar estilos más autoritarios, orientados a la tarea y transaccionales, mientras que las mujeres exhiben estilos más democráticos, interactivos u orientados a las relaciones, y transformacionales (Eagly y Johannesen-Schmidt). Actualmente existe un considerable volumen de trabajos que tienden a revalorizar «lo femenino» en contextos organizativos que tradicionalmente han operado sobre asunciones y patrones de comportamiento típicamente masculinos. Se trata de un discurso que tiende a ganar popularidad en círculos empresariales, educativos y, hasta cierto punto, en el sentido común, y que reclama la celebración de los valores femeninos y la valoración de la diversidad en la coyuntura actual de la gestión empresarial. Este discurso es convergente -en apariencia- con los «nuevos» y más suaves discursos sobre gestión que se centran en la buena gestión de las personas como la nueva fuente de productividad en las organizaciones flexibles posmodernas. Los textos de gestión -fundamentalmente los destinados a un público no académico- hablan, en este sentido, de la «ventaja femenina» en el liderazgo.

De acuerdo con la autora feminista (Helgesen), los talentos, las experiencias y las destrezas que poseen las mujeres son precisamente lo que se necesita para el liderazgo en la economía postindustrial, lo que está creando oportunidades sin precedentes para que las mujeres ocupen un rol crucial en liderar el cambio transformacional en organizaciones y comunidades. Desde esta perspectiva, por tanto, la presencia de las mujeres en puestos de responsabilidad se justifica por su capacidad de desarrollar estilos de liderazgo de acuerdo con los nuevos modelos organizativos «postmodernos» centrados en las relaciones horizontales y el trabajo en equipo. Las aproximaciones que acabamos de considerar al abordar las relaciones entre género y liderazgo ofrecen posibilidades y abren vías de análisis que permiten redirigir nuestra atención hacia cuestiones tradicionalmente silenciadas en el estudio de las instituciones. Sin embargo, presentan también limitaciones que restringen las posibilidades de ofrecer alternativas a los discursos dominantes. El enfoque igualitario, por 
un lado, centrado en erradicar los estereotipos de sexo y afirmar la igualdad esencial entre hombres y mujeres, ha tendido a soslayar el significado socialmente construido del género, y se ha traducido comúnmente en una estrategia de asimilación de los modelos establecidos que no cuestiona las relaciones de poder existentes. Por su parte, el enfoque centrado en celebrar la diferencia femenina, ampliamente reconocido en los planteamientos actuales sobre el liderazgo, socava las posibilidades de pensar sobre el cambio en las relaciones de género al distraer la atención de las relaciones de género/poder imbricadas en la vida organizativa, los valores, las estructuras y los procesos. Se trata, además, de un discurso fácilmente cooptable por las lógicas dominantes de la gestión empresarial.

Para concluir este apartado nos vienen muy bien las palabras de Nájera «esas dos modalidades tradicionales del feminismo están agotadas y se hace necesario explorar un camino alternativo que se aleje de esencialismos y que ha de desbordar forzosamente categorizaciones poco flexibles».

\subsection{El liderazgo de equipos de investigación}

Uno de los indicadores de éxito de la carrera investigadora de las académicas es que dirijan equipos de investigación puesto que ello significa, a grandes rasgos, que tienen un reconocimiento como investigadoras a nivel de la comunidad científica local e internacional.

En los últimos años algunas aproximaciones a la investigación desde la perspectiva de género han ayudado a esclarecer como mínimo la distribución por sexos del personal investigador (Pérez-Sedeño; Miqueo, Barral y Magallón), así como las categorías profesionales que ocupan globalmente las mujeres y hombres aunque hasta el momento carecemos de datos mínimamente fiables sobre la productividad e impacto de su trabajo.

Efectivamente, y así lo expresa el informe MEC: Académicas en cifras 2007 que pretende servir de instrumento de trabajo para conocer la situación real de las mujeres en el sistema universitario español: «Ellas son mayoría entre los titulados en la universidad pública (60\% en el curso 2005-2006), mientras que el número de catedráticas no alcanza el 14\%».

Las investigaciones que se han llevado a cabo en el tema, hasta el momento contemplan tópicos tales como los roles académicos y el género, los obstáculos que las mujeres investigadoras tienen en su carrera y los factores que contribuyen al éxito.

Se presentan a continuación las principales conclusiones realizadas en el ámbito universitario sobre las diferencias otorgadas al género: 
- Existe una relación tradicional entre lo masculino y las ciencias. La Academia se percibe de manera tradicional como elitista, masculina y patriarcal (Asmar; Poole y Langan-Fox; Rutherford) a pesar de que en los últimos años se han experimentado progresos, estas percepciones siguen estando presentes (Amancio; Asmar; Bagilhole).

- La literatura pone de manifiesto que, a pesar de que se han hecho progresos en la participación de las mujeres en la Educación Superior, todavía hay diferencias de género en cuanto a los roles que tienen en las universidades (Sagaria y Agans; Saunderson;Smeby y Try).

- La presencia de las mujeres en la gestión universitaria y en los núcleos de poder es claramente minoritaria. Los escalones superiores siguen dominados por hombres (Bagilhole; Rutherford)

- Las universidades son «bastiones» de los hombres y la discriminación aún existe en forma de redes, especialmente de investigación (Kyvik y Teigen; Webster)

- Las mujeres tienen menos productividad investigadora que los hombres (Olssen y Peters)

Sintetizamos a su vez los obstáculos que las mujeres investigadoras tienen en su carrera en la revisión de estudios realizada:

- La división de género en la actividad académica (las mujeres se dedican más a la docencia y los hombres más a la investigación (Bagilhole y White; Park).

- Las mujeres tiene menos confianza en sus habilidades y menos acceso a las redes académicas (Britton; Dean, Johnson, Jones y Lengkeek; Doherty y Manfredi).

- La existencia de presiones entre la vida personal y la profesional (Forster; Probert).

- La falta de redes y de trabajo colaborativo que provoca que el acceso a la financiación sea reducido (Lafferty y Fleming).

- Las mujeres obtienen menos financiación, menos recursos y tienen menos ayudantes de investigación (Toren).

- En ciencias sociales y humanidades la cultura organizativa es tradicionalmente más individualista (Asmar).

- Las mujeres producen menos publicaciones y esto genera que haya menos financiación para sus proyectos- el ciclo de la productividad de la investigación- (Soliman y Soliman). 
La investigación dedicada al estudio de los factores de éxito de las profesoras que han llegado a cierto grado de reconocimiento como investigadoras en sus áreas de conocimiento se ha orientado a detectar factores tales como:

- El análisis de los programas de mentoring en los que pueden participar las mujeres para mejorar sus capacidades investigadoras.

- El análisis de la formación recibida por las investigadoras a nivel formal e informal.

- El análisis de las estrategias, las culturas de trabajo y los contextos asociados al éxito (Dever y Morrison).

- La flexibilidad asociada al nivel de autonomía y la capacidad de gestionar el tiempo de forma efectiva.

- Se analiza la satisfacción asociada al trabajo investigador. Las mujeres perciben sus condiciones de trabajo como más satisfactorias en términos de satisfacción «intrínseco-subjetiva» frente a los hombres que se dejan conducir por motivación «extrínseca-objetiva» como: estatus, sueldo, condiciones de trabajo (Poole y Langan-Fox).

\subsection{La participación de las mujeres en los órganos de gobierno o comisiones delegadas}

A pesar de que la participación femenina en los órganos de gobierno universitarios es una cuestión poco explorada por la investigación, sí se dispone de todo un cuerpo de estudios que, bajo la rúbrica general de «mujeres en la gestión» (women-in-management), se ha ocupado de analizar el acceso de la mujer a las posiciones más altas dentro de las estructuras organizativas existentes y de contribuir a la ruptura del «techo de cristal» que impide a muchas mujeres participar en la gestión de las organizaciones.

Aunque como se ha dicho anteriormente no existen muchos trabajos que exploren la participación de la mujer en procesos de toma de decisiones en el seno de los órganos de gobierno de la Universidad, sí se dispone, en cambio, de un amplio abanico de estudios que abordan el papel del género en la interacción grupal durante la toma de decisiones.

La participación de la mujer en los órganos de gobierno no hay que justificarla sólo por cuestiones técnicas o instrumentales, sino también por razones de democracia participativa. La cuestión de la representación de la mujer en los diversos órganos de gobierno de nuestra sociedad constituye el tema central del trabajo de Phillips: «Engendering democracy», donde se explora la intersección entre la teoría feminista y la teoría democrática. En abierto contraste con la 
teoría democrática más tradicional, que presupone un concepto de ciudadanía abstracto y donde el género está ausente, Phillips sostiene que la auténtica igualdad entre hombres y mujeres sólo puede conseguirse si se reconocen las diferencias de género. En cambio, para Phillips, tal reconocimiento constituye sólo una fase necesaria, aunque transitoria, hacia una sociedad en la que el género llegue a ser irrelevante. Para que esta transición hacia una sociedad en la que el género es irrelevante tenga lugar, las mujeres deben convertirse primero en participantes plenas de la vida política, entendida en sentido amplio. Un argumento similar puede extrapolarse al ámbito de la gestión y participación universitarias.

A pesar de que en su conjunto la investigación sobre comportamiento comunicativo no consigue identificar diferencias tajantes y replicables en función del género, sí existe mayor acuerdo en afirmar que, lejos de ser un elemento neutral, el género ejerce una influencia considerable en los procesos de toma de decisiones. En concreto, podemos agrupar estos trabajos en tres grandes ámbitos de estudio que ponen en relación la toma de decisiones con el género: (1) el interesado propiamente en las diferencias conductuales en función del género; (2) el preocupado por la capacidad de influencia asociada al género durante la toma de decisiones, y (3) el orientado a determinar los efectos de la composición del grupo en el proceso de toma de decisiones.

En cuanto a las diferencias en los comportamientos grupales en función del género, la literatura apunta -aunque no de forma concluyente- ciertas diferencias de género en la comunicación. Un rasgo distintivo en el estilo conversacional femenino es el mantenimiento de la conversación, orientado a establecer conexión con los demás a través del diálogo. De manera similar, plantear cuestiones es un medio para estimular la inclusión y la conexión durante la comunicación grupal, valores éstos distintivamente femeninos. Parece, en este sentido, que esta preocupación de las mujeres por la conexión las conduce a plantear cuestiones a sus compañeros para incluirlos en la interacción grupal. En síntesis, las mujeres valoran la conexión, la cooperación y la expresión de las emociones más que los hombres. Sin embargo, pueden existir ciertas diferencias de género en cuanto al modo en que se formulan las preguntas.

El trabajo de Hawkins y Power aborda directamente esta última cuestión, resultando de especial interés para esta investigación tanto por los problemas que plantea como por la metodología empleada. Su estudio analiza la interacción en 18 pequeños grupos de toma de decisiones para responder a la pregunta de si existen diferencias de género en cuanto al número y contenido de las 
cuestiones realizadas en contextos grupales pequeños de toma de decisiones. Para el análisis de esta cuestión plantean el uso de la escala de Bales, que se reconoce como la taxonomía más utilizada en la investigación interesada por el análisis de la interacción dentro de grupos pequeños. Sin embargo, se reconoce la utilidad de esta escala para capturar las funciones más importantes de las preguntas dentro de la toma de decisiones dentro de grupos pequeños, en los que el intercambio de información es crucial. La escala de Bales está complementada con otra taxonomía que evalúa el grado de apertura de la interacción personal. En su conjunto, el instrumento observacional empleado se muestra útil para analizar las diferencias de género en la realización de preguntas, ya que algunas investigaciones indican que los hombres tratan de controlar la introducción y el desarrollo de los tópicos más que las mujeres.

Los resultados de la investigación de Hawkins y Power indican que las preguntas juegan un papel importante en la toma de decisiones dentro de los pequeños grupos y que el género constituye un factor relevante en su producción. Aunque hombres y mujeres presentan una probabilidad similar de plantear preguntas, sí es posible identificar diferencias significativas en el tipo de preguntas que se realizan durante el proceso de toma de decisiones. Así, las mujeres presentan una probabilidad mayor de realizar preguntas de tipo exploratorio que sus compañeros hombres. Este tipo de preguntas invitan a la elaboración de argumentos, a compartir la información y la opinión. Aunque algunos trabajos pueden relacionar este tipo de comportamiento comunicativo con la inseguridad y la necesidad de autoafirmación, Hawkins y Power lo vinculan a un doble objetivo: estimular la implicación en la interacción y la participación, y obtener detalles de la información necesaria para hacer mover el grupo hacia la consecución de su objetivo. En este sentido, se puede argumentar el impacto de la participación femenina en la eficacia de la toma de decisiones grupales, en tanto que sirve al doble propósito de obtener el detalle necesario para servir de base a una decisión eficaz e implicar al grupo en una evaluación crítica de sus propias asunciones y opiniones, desvelando así informaciones falsas o razonamientos erróneos que podrían conducir a decisiones de baja calidad.

Otras investigaciones apoyan esta idea de que comportamientos como el fomentar la participación de otros y buscar visiones opuestas guardan relación con la percepción de eficacia y calidad en la toma de decisiones por parte del grupo. Mayer, por ejemplo, plantea que comportamientos tales como estimular a los miembros a participar, buscar visiones opuestas y presentar evidencia de las sugerencias se relacionan con la percepción de calidad en la toma de 
decisiones. Similarmente, los comportamientos socioemocionales negativos se perciben disfuncionales para la eficacia de la decisión, mientras que los positivos la mejoran.

Otras investigaciones, centradas en el análisis de los procesos de negociación, ponen de manifiesto que los estereotipos de género favorecen la construcción del «buen negociador» en términos preferentemente masculinos. Así, el trabajo de Kray y otros identifican la asertividad, la capacidad de solución de problemas, la consideración de los propios intereses y el conocimiento como atributos típicamente masculinos que tienden a configurar una «ventaja masculina» durante las negociaciones grupales.

Con independencia de las causas, un buen número de trabajos parten de la tesis de que las personas, al interactuar en procesos grupales de toma de decisiones, perciben diferencias entre los participantes en función del género. Balwell y Berger, por ejemplo, determinan que el papel del género en la interacción cara a cara puede venir determinado por distintas concepciones que usan los participantes, pudiéndose detectar diferencias en cuanto a la cantidad de tiempo que se está hablando, la frecuencia de los gestos, las estructuras de iniciación de la conversación, o la fijación de la mirada mientras se habla o escucha. Similarmente, Smith-Lovin y Brody analizan las estructuras de interrupción como factor relativo a la calidad en la toma de decisiones, concluyendo que los hombres tienen mayor tendencia a interrumpir las mujeres y que la composición del grupo se configura como uno de los elementos más relevantes para explicar los procesos de interacción.

En cuanto a la capacidad de influencia durante la toma de decisiones, las investigaciones tienden a resaltar la menor influencia femenina como consecuencia del estereotipo social de menor competencia instrumental que suele atribuirse a la mujer. Algunos trabajos, sin embargo, matizan esta afirmación aludiendo a determinados rasgos propios del estilo femenino de toma de decisiones que permiten moderar la incidencia de dichos estereotipos, como puede ser la demostración de destrezas específicas relacionadas con la tarea, la motivación hacia la cooperación, y la atención a soluciones de alta calidad (Shackelford, Wood y Worchel). Carli señala que las mujeres son menos influyentes cuando utilizan formas dominantes de comunicación, mientras que los hombres ejercen menor influencia en dominios tradicionalmente asociados al rol femenino o en grupos que cuentan con una mayor presencia femenina. Asimismo sugiere que los hombres tienden a resistir más la influencia ejercida por parte de las mujeres, especialmente cuando éstas hacen uso de estilos de comunicación altamente competentes, aunque esta resistencia puede verse 
reducida cuando las mujeres equilibran su competencia en muestras de calidez y orientación comunitaria. En definitiva, pues, estos trabajos muestran que, a pesar de que los estereotipos femeninos funcionan restando protagonismo a la mujer en los procesos grupales, algunos de los rasgos que suelen atribuirse a un estilo femenino de toma de decisiones, en presencia de determinados factores contextuales y grupales, contribuyen a moderar el efecto negativo de tales estereotipos.

Finalmente, en cuanto a los efectos de la composición del grupo en el proceso de toma de decisiones, las investigaciones coinciden en señalar el papel moderador que la característica del grupo (tamaño, proporción de hombres y mujeres,...) tiene sobre el género y su influencia en la interacción. Lépine y otros, por ejemplo, reexaminan las conclusiones sobre la superioridad de los equipos masculinos en tareas tradicionalmente consideradas masculinas, e ilustran cómo, en algunos contextos, los equipos de toma de decisiones dominados por hombres pueden constituir la peor composición de género. Además, en su trabajo también incluyen que a medida que el porcentaje de hombres se incrementa, aumenta exponencialmente la tendencia a tomar decisiones de tipo agresivo. Otros trabajos, en cambio, inciden en que los estudios sobre interacción entre iguales con similar poder y estatus muestran pocas diferencias de género en el comportamiento, y que la mayoría de las interacciones entre hombres y mujeres ocurren en el contexto estructural de roles o relaciones de estatus que son desiguales. Son estas diferencias de poder y estatus que a menudo se confunden con el género en sí mismo las que crean efectos reales en la interacción (Ridgeway y Smith-Lovin).

\section{METODOLOGÍA}

Se presenta en este artículo una síntesis de tres investigaciones sobre el liderazgo y la participación en los órganos de gobierno de la Universidad con el objetivo de contribuir a averiguar las razones por las cuáles en la Universidad persisten diferencias en función del género del profesorado.

Presentamos a groso modo los objetivos y metodologías seguidas en dichas investigaciones en la tabla 1. 
Tabla 1. Resumen de objetivos y metodologías sobre liderazgo, participación y género en la Universidad de investigaciones dirigidas por Marina Tomàs

\begin{tabular}{|c|c|c|c|}
\hline $\begin{array}{l}\text { Proyecto de Investigación } \\
\text { Personas equipo } \\
\text { Institución subvencionadora }\end{array}$ & Objetivos & $\begin{array}{l}\text { Instrumentos } \\
\text { y técnicas de } \\
\text { recogida y análisis } \\
\text { de información }\end{array}$ & Informantes \\
\hline $\begin{array}{l}\text { La perspectiva del género en } \\
\text { las universidades y el ejercicio } \\
\text { de cargos académicos. } \\
\text { Tomàs, M (Coord.); Durán, } \\
\text { M.M; Guillamón, C. y Lavié, } \\
\text { J.M. } \\
\text { ICD, } 2002\end{array}$ & $\begin{array}{l}\text { 1. Conocer la } \\
\text { percepción del } \\
\text { profesorado sobre } \\
\text { el liderazgo que } \\
\text { ejercen las mujeres } \\
\text { cuando dirigen sus } \\
\text { departamentos } \\
\text { 2. Obtener evidencias } \\
\text { de la forma de liderar } \\
\text { de las mujeres para } \\
\text { contribuir a discernir } \\
\text { si existen estilos de } \\
\text { liderazgo en función } \\
\text { del género. }\end{array}$ & $\begin{array}{l}\text { - Cuestionario al } \\
\text { profesorado cuyo } \\
\text { departamento es } \\
\text { dirigido por una } \\
\text { directora. } \\
\text { - Entrevistas a } \\
\text { directoras. }\end{array}$ & $\begin{array}{l}150 \\
\text { cuestionarios } \\
\text { profesorado } \\
18 \text { entrevistas }\end{array}$ \\
\hline $\begin{array}{l}\text { Estudio de las dinámicas en } \\
\text { el seno de los órganos de } \\
\text { gobierno de las universidades } \\
\text { desde la perspectiva de género. } \\
\text { Tomàs, M (Coord.) } \\
\text { Duran,M.M; Feixas,M; } \\
\text { Guillamón,C. y Lavié,J.M } \\
\text { AGAUR, } 2007\end{array}$ & $\begin{array}{l}\text { 3. Observar el tipo de } \\
\text { participación en los } \\
\text { órganos colegiados } \\
\text { en donde se toman } \\
\text { decisiones en función } \\
\text { del género. }\end{array}$ & $\begin{array}{l}\text {-Observación de } \\
\text { scripts y roles } \\
\text { en reuniones de } \\
\text { distintos órganos } \\
\text { de gobierno y } \\
\text { focusgroups. }\end{array}$ & $\begin{array}{l}8 \text { órganos de } \\
\text { gobierno } \\
\text { (199 personas } \\
\text { observadas de las } \\
\text { cuales } 97 \text { eran } \\
\text { mujeres y } 102 \\
\text { hombres) } \\
2 \text { focusgroup } \\
\text { (un total de } 16 \\
\text { expertos) }\end{array}$ \\
\hline $\begin{array}{l}\text { La investigación académica de } \\
\text { las investigadoras en Ciencias } \\
\text { Sociales en las universidades } \\
\text { UAB y UB. } \\
\text { Tomàs,M (Coord.) } \\
\text { Bernabeu,D.; } \\
\text { Castro,D; } \\
\text { Duran,M.M.; } \\
\text { Ion,G; } \\
\text { Vlachopoulos,D. y } \\
\text { Mentado,T. } \\
\text { ICD. Ref. U-45/10 }\end{array}$ & $\begin{array}{l}\text { 4. Conocer el perfil } \\
\text { de las investigadoras } \\
\text { que dirigen grupos } \\
\text { de investigación de } \\
\text { prestigio. }\end{array}$ & $\begin{array}{l}\text {-Entrevistas } \\
\text { focalizadas a nivel } \\
\text { individual. } \\
\text {-Análisis del } \\
\text { discurso en } \\
\text { la producción } \\
\text { científica. }\end{array}$ & 14 entrevistas \\
\hline
\end{tabular}


Se han llevado a cabo todas ellas en las universidades metropolitanas de Barcelona.

Respecto al objetivo 1 y 2 de la primera investigación se elaboró un cuestionario ad hoc a fin de averiguar las oportunidades de desarrollo profesional de las directoras de departamentos universitarios y los atributos que se otorgaban al liderazgo femenino.

En cuanto a las entrevistas se diseñó un guión dividido en tres bloques: datos biográficos de la entrevistada, contexto de desempeño del cargo y, finalmente, el ejercicio de dirección y las preferencias de liderazgo.

Respecto al objetivo 3 correspondiente a la segunda investigación presentada se realizaron ocho observaciones a diferentes órganos de gobierno de dos universidades tales como: Comisión de investigación, 4 Consejos de departamento (de diferentes áreas de conocimiento), 2 Juntas permanentes y 1 Comisión académica. Tres de ellas estaban dirigidas por mujeres y cinco por hombres. Mientras que las profesoras han dirigido un grupo pequeño, uno mediano y uno grande, en el caso de sus compañeros, cuatro han dirigido un grupo de tamaño mediano y uno, un grupo de tamaño grande.

En las observaciones se siguió la teoría de la interacción de R. F. Bales (Vendrell). Esta aproximación entiende los grupos como sistemas de individuos que interactúan. La actividad del grupo se concibe como una secuencia de acciones y reacciones que se consideran interacciones. Concretamente, la interacción en un grupo se divide en un conjunto de actos microscópicos. Un acto es una comunicación o una manifestación expresiva, verbal o no verbal, que en su contexto puede ser entendida por otro miembro como equivalente a una frase sencilla. Para Bales, todas las acciones del grupo se consideran interacciones y se pueden analizar mediante la observación sistemática.

Para las observaciones se han definido 8 categorías, de las cuales cuatro se consideran roles (Rol iniciador, Rol co-iniciador, Rol opositor, Rol seguidor) y cuatro, scripts (Script asertivo, Script no asertivo, Script agresivo y Script «de huida»).

Respecto al objetivo 4 correspondiente a la tercera investigación presentada, en cuanto a las entrevistas se diseñó un guión que comprendía los siguientes aspectos: antecedentes y contexto actual del profesional, el rol de las personas que investigan y de las otras funciones académicas, factores de éxito, liderazgo de grupo de investigación y difusión del conocimiento y de la investigación. 


\section{RESULTADOS}

Se aporta una síntesis de los resultados de cada una de las investigaciones que tienen que ver con los factores de la Universidad que impiden esta igualdad de oportunidades. Es decir es una selección transversal de resultados en base a este propósito.

\subsection{Factores relativos al liderazgo en relación a la igualdad de oportunidades de género en la Universidad}

Se identifica la existencia de dificultades tanto externas a la propia mujer, como internas a ella, que nos indican posibles explicaciones sobre la escasa presencia del género femenino en la gestión universitaria. Entre las dificultades externas, la cultura organizativa de la Universidad aparece como un elemento resistente al cambio que fomenta un entorno predominantemente dominado por valores masculinos y que puede justificar la auto-exclusión de algunas mujeres de la gestión universitaria. Asimismo, se identifica los principales atributos con los que las mujeres participantes describen su propio rol en la gestión y como creen que éste contribuye a un liderazgo eficaz en la Universidad. Por un lado las oportunidades de desarrollo profesional que las directivas manifiestan haber tenido en sus carreras profesionales universitarias así como las destrezas y los atributos de liderazgo que las personas de la comunidad universitaria consultadas asignan a sus directivas y directivos. Por otra parte, las experiencias de gestión de las mujeres gestoras en el puesto de trabajo: barreras que han encontrado, percepciones experimentadas en torno a su rol y destrezas de liderazgo.

Se ha observado la presencia de una serie de rasgos de liderazgo en las mujeres que ejercen cargos académicos en la Universidad así como de interés de los liderados por el tipo de liderazgo presente en su directora de departamento. Sin ánimo de comparación y a título orientativo se han extraído los siguientes rasgos. En la tabla n. ${ }^{\circ} 2$ se presenta en la columna de la izquierda (a) las características que creían que deberían tener sus directoras de departamento y en la columna de la derecha (b) los rasgos y características más frecuentes en sus directoras de departamento. 
Tabla 2. Características que deberían tener (a) y que tienen (b) las directoras de departamentos universitarios (Guillamón, Cristina y Marina Tomàs, 2002)

\begin{tabular}{|c|c|}
\hline $\begin{array}{l}\text { Características que deberían tener las } \\
\text { directoras de departamento (a) }\end{array}$ & $\begin{array}{l}\text { Características presentes en sus } \\
\text { directoras de departamento (b) }\end{array}$ \\
\hline - Tener espíritu de ambición & - Ser responsable \\
\hline - Ser una persona competitiva & - Ser resistente a los frustraciones \\
\hline - Ser resistente a las frustraciones & - Ser asertivo/va \\
\hline - Actuar como persona dialogante & - Ser diplomático/a \\
\hline - Tener habilidades comunicativas & $\begin{array}{l}\text { - Capacidad por pensar y hacer diversas } \\
\text { cosas a la vez }\end{array}$ \\
\hline $\begin{array}{l}\text { - Ejercer un liderazgo de estilo } \\
\text { democrático }\end{array}$ & $\begin{array}{l}\text { - Poner atención en las relaciones } \\
\text { personales }\end{array}$ \\
\hline \begin{tabular}{|l} 
- Preocupación por el propio desarrollo \\
profesional
\end{tabular} & $\begin{array}{l}\text { - Actuar como persona dialogante y de } \\
\text { consenso }\end{array}$ \\
\hline \multirow{3}{*}{$\begin{array}{l}\text { - Gestionar con eficacia y eficiencia los } \\
\text { recursos del departamento }\end{array}$} & - Tener habilidades comunicativas \\
\hline & $\begin{array}{l}\text { - Gestionar con eficacia y eficiencia los } \\
\text { recursos del departamento }\end{array}$ \\
\hline & $\begin{array}{l}\text { - Capacidad por obtener recursos } \\
\text { económicos para el departamento }\end{array}$ \\
\hline
\end{tabular}

Entre las barreras que impiden a las profesoras universitarias ejercer cargos de gestión encontramos algunas de tipología interna y otras de tipología externa. Entre las internas se pueden citar las propias de los procesos de socialización: diferencias en la educación de niños y niñas, miedo a defraudar las expectativas del papel femenino, falta de modelos donde mirarse, poco interés por el estilo de liderazgo tradicional o baja autoestima. Entre las externas podemos citar la cooptación, la dificultad de conciliación entre vida profesional y familiar, la cultura sexuada de las universidades y los estereotipos ligados al género femenino y el liderazgo.

Las participantes en nuestra investigación afirman no sentirse a gusto con los valores y las estrategias de acción existentes en los órganos de poder de sus universidades por el hecho de ser claramente ajenos a ellas en tanto que no han participado en su generación. Esto ocasiona poca vinculación a la institución y poco deseo de liderarla. El rechazo a los modelos tradicionales de liderazgo imperantes las desmotiva para postularse como candidatas al ejercicio de cargos. 
Respecto las percepciones de las directivas sobre su propio comportamiento de liderazgo y los efectos que éste produce, las respuestas de las participantes no son unánimes. Algunas niegan las diferencias en función de género y las atribuyen exclusivamente a la individualidad de cada cual y mencionan la necesidad de considerar otras variables además del género al estudiar el tema del liderazgo. Otras reconocen un discurso popular que atribuye a las mujeres un modo de liderar más participativo y basado en la comunicación interpersonal.

Ahora bien, en lo que sí coinciden todas las participantes es en afirmar que ocupar un lugar de poder sirve para «poder hacer algo, poder cambiar algo». Se desprecia el «poder sobre alguien» pero se aprecia el «poder para algo»y el «poder con alguien». Ello nos lleva a considerar las destrezas y habilidades que las participantes atribuyen a las mujeres que ejercen cargos directivos.

Uno de los principales rasgos que se les reconocen a las mujeres que lideran es su capacidad para controlar la realización de los programas ("poder para»), seguido de la capacidad para desburocratizar los procedimientos y atender a las necesidades de las personas lideradas y el contar con éstas para el ejercicio del cargo («poder con»). El trabajo en equipo y la preocupación por conseguir un clima de trabajo positivo, son cualidades que las participantes en el estudio atribuyen a las mujeres líderes.

Las entrevistadas han mostrado, en su mayoría, haber estado sujetas a procesos de evaluación y promoción construidos en referencia a una norma 'masculina' que las discriminaba abiertamente.

\subsection{Factores relativos al liderazgo de equipos de investigación en relación a la igualdad de oportunidades de género en la Universidad}

El análisis de los grupos de investigación consolidados nos ayuda a aproximarnos al campo del estudio de las élites académicas. Se trata de académicos y académicas que han llegado a un alto nivel de desarrollo de su carrera profesional investigadora. Como apuntan otros estudios (ver por ejemplo García de León) el estudio de las elites forma parte de la sociología del poder, tema substancial en ciencias sociales y llama la atención sobre cómo se distribuye el poder en la ciencia. Las elites son la «punta del iceberg» de los procesos de cambio aunque la Universidad actual catalana ha conocido en los últimos años cambios importantes, este proceso no va acompañado por un cambio en la distribución igualitaria entre hombres y mujeres en el ámbito de la investigación en general y en ciencias sociales en especial.

En la distribución por género de los equipos de investigación consolidados, en casi todos los casos, el número de grupos liderados por hombres es superior al número de grupos liderados por mujeres. Concretamente, del total de 
grupos reconocidos (1078) por la Generalitat de Catalunya en el 2007, solo unos 241 están liderados por mujeres frente a 847 que están liderados por hombres, cosa que en porcentajes representa que sólo un $27,6 \%$ de los grupos están coordinados por investigadoras.

Con el objetivo de conocer los elementos que favorecen y que dificultan la producción científica de las mujeres en la Universidad se identifica como una dificultad el hecho mismo de ser mujer, dado que en la cultura universitaria todavía se consideran ciertas actividades como más propias del género masculino que del femenino, por ejemplo la organización de congresos científicos. En este sentido se ve la conveniencia de hacer redes entre las mujeres para darse soporte mutuo.

Lo que se puede afirmar de lo que piensan las investigadoras que dirigen grupos consolidados es lo siguiente:

- El tiempo dedicado a la gestión más bien preocupa que no interesa a las investigadoras. En general las entrevistadas lo consideran 'un mal necesario'.

- Valoran de forma muy positiva las estancias de investigación y estudio en universidades extranjeras así como la posibilidad de tener relaciones con personal académico de otros países. Dan una importancia especial a la posibilidad de colaborar en redes de trabajo internacional en donde ellas participen por su expertez.

- Las investigadoras que lideran grupos son partidarias de delegar tareas y responsabilidades en los miembros de sus equipos. Consideran que deben procurar un clima del grupo positivo en el que trabajar de forma agradable.

- Todas las investigadoras coinciden en la necesidad de distribuir el liderazgo puesto que favorece la producción científica.

- El establecer normas de funcionamiento claras y compartidas por todos los miembros del grupo es una preocupación común entre las entrevistadas.

- Un buen clima es necesario para asegurar la creación de conocimiento que comporta una cultura de colaboración entre los miembros.

- La autoevaluación del equipo de trabajo se considera muy importante para la mejora de la actividad investigadora.

- Parece que el hecho de ser hombre o mujer, condiciona el tipo de tareas que se asumen dentro del grupo de investigación, de acuerdo con parámetros socialmente aceptados desde un modelo masculino. 
3.3. Factores relativos a los roles en la participación en relación a la igualdad de oportunidades de género en la Universidad

Una de las primeras cuestiones que ocupan el debate sobre las relaciones entre órganos de gobierno en la Universidad y género es la falta de presencia de mujeres en los cargos de gestión, especialmente en los de más alto nivel. La presencia de las mujeres en los órganos de gestión se justifica, en cualquier caso, tanto por cuestiones de justicia e igualdad social como por motivos de eficiencia y calidad en la toma de decisiones.

Se constata que, aparte de la creciente incorporación de la mujer a la educación universitaria e, incluso, la feminización de determinadas áreas de conocimiento, la representación femenina en los órganos de gobierno universitarios sigue siendo sensiblemente inferior en comparación con la población masculina. En este sentido, la Universidad se sitúa detrás de otras organizaciones en el desarrollo e implantación de políticas de igualdad de género.

Si bien el género no puede convertirse en una categoría absoluta para la explicación de todas las diferencias en los modos y estilos de participación, la influencia del género es incuestionable. Además de los factores de orden intrapsíquico y educativos, la clase social genera también su propio código relacional.

Es importante señalar el papel del contexto como moderador de estas posibles diferencias vinculadas al género. Por un lado, pensar en la Universidad como contexto y de actuación implica asumir que todos los miembros de la comunidad universitaria, hombres y mujeres, han sido sometidos a un proceso de socialización similar derivado del entorno académico que conduce a la masculinización.

Por otra parte, no es lo mismo hablar de diferencias relativas al estilo de gestión o liderazgo, que de aquellas que hacen referencia a los estilos de participación o intervención en los foros de toma de decisiones. En este último caso, las diferencias hombre/mujer parecen ser evidentes, a pesar de que el contexto (micro) grupal ejerza una influencia considerable en la definición de las pautas de intervención.

Teniendo en cuenta todas estas precisiones en torno a las diferencias entre estilos femeninos y masculinos, y la importancia que en su definición tienen los factores contextuales y grupales, los argumentos debatidos en los focusgroup establecen una caracterización de lo que podríamos denominar una «clave femenina» de participación en la toma de decisiones que se sintetiza, en primer lugar, por exhibir un estilo directo y conciso en cuanto a la participación. Es decir, las mujeres tienden a realizar menos intervenciones públicas, y éstas, en cuanto las realizan, suelen tener un carácter más concreto y resolutivo, en el 
sentido de que suelen estar más orientadas a la resolución del problema que se discute y estar fundamentadas en «razonamientos más consistentes».

Otra característica de este estilo femenino de participación se relaciona con el mayor pragmatismo que las mujeres suelen mostrar en sus intervenciones. Esta actitud pragmática que adoptan muchas mujeres en la toma de decisiones suele traducirse en la relatividad de las ideas geniales y brillantes. El estilo femenino de toma de decisiones puede caracterizarse, pues, como más realista en la consideración de las diferentes posibilidades de actuación y, por tanto, en tonalidad más conservadora.

Forma parte de esta sensibilidad femenina una atención a los aspectos relativos al proceso y ocupar «el espacio fronterizo». Esta atención al proceso se relaciona, por un lado, con una preocupación por el desarrollo de la reunión y de la dinámica del grupo pero también tiene relación con una concepción más amplia sobre el trabajo en grupo y los elementos que pueden optimizar su funcionamiento. En este sentido, la distinción tradicional que caracteriza el liderazgo masculino como «orientado a la tarea» y el liderazgo femenino como «orientado a las relaciones», puede reproducirse en lo que se refiere a los modos de intervención y participación en los procesos grupales de toma de decisiones. Las mujeres, en general, muestran una mayor preocupación por crear «una determinada atmósfera» que facilite el proceso de trabajo colectivo.

Las causas que explican las diferencias se podrían agrupar según se ponga el énfasis en la participación e implicación de las mujeres en los órganos de gobierno, en el interés por acceder a los órganos de gobierno, o atribuibles al grado de satisfacción en la toma de decisiones en los órganos de gobierno.

De algún modo las diferencias existentes en la participación entre hombres y mujeres en los órganos de gobierno pueden depender de un conjunto de causas como tipología de contexto (reuniones a nivel de departamento, facultad, rectorado,...), composición del grupo (tamaño, género,...) y características personales (categoría profesional, status, estado civil,...).

Respeto al número de intervenciones clasificadas en scripts no se aprecian diferencias respecto al género. Sólo en el caso de intervenciones con script agresivo, que parecen patrimonio del género masculino.

Con respecto a los roles se observa una tendencia a que el género masculino sea más iniciador y juegue un rol de opositor con mayor frecuencia. Por otra parte quedan más representados por el género femenino los roles de co-iniciadora y seguidora.

A pesar de que los resultados no son concluyentes, y deben ser matizados en cualquier caso por la influencia de variables contextuales y personales, es posible identificar una serie de comportamientos diferenciales en los 
procesos de toma de decisiones asociados al género de los participantes como la estructura de iniciación-participación, la estructura de interrupciones, los comportamientos no verbales exhibidos, o la taxonomía de preguntas que se realizan durante el proceso de toma de decisiones. Igualmente, es posible identificar una serie de variables moderadoras del papel del género en los procesos de interacción grupal, como la composición del grupo, el tipo de tareas a realizar y las decisiones que se deben adoptar.

Más que el género per se, hay una serie de factores relacionados con el género que ejercen una influencia considerable en los procesos de interacción grupal, como el poder y el estatus de los participantes, los estereotipos asociados al género, etc. y los estereotipos sociales de género ejercen influencia a la hora de conceder una cierta «ventaja masculina» en la toma de decisiones.

\section{DISCUSIÓN Y CONCLUSIONES}

La incorporación de la mujer en la gestión universitaria se justifica tanto por razones de justicia e igualdad social como por motivos de eficiencia y calidad en la toma de decisiones.

La Universidad no es pionera en la asunción y representación de cargos de gestión por parte de las mujeres. A pesar de la creciente presencia de la mujer en los órganos de gobierno universitarios, la figuración femenina sigue siendo menor que la masculina.

Los motivos que pueden explicar esta menor presencia de la mujer en la gestión universitaria son múltiples y se relacionan tanto con factores estructurales como culturales. La categoría profesional y la edad, la experiencia en gestión, las responsabilidades familiares o atención a terceras personas son algunas de las causas que explican la menor participación de las mujeres en los órganos de gobierno.

El género, como aspecto constitutivo de las relaciones interpersonales, a veces está presente de manera tácita en las dinámicas que se generan en el interior de los órganos de gobierno, lo que invita a reconsiderar cualquier definición de calidad en la toma de decisiones en términos puramente técnicos.

Aunque la influencia del género en las formas y estilos de participación resulta incuestionable, éste no puede convertirse en la única explicación de las diferencias de comportamiento perceptibles en los procesos de participación y toma de decisiones.

Es posible identificar una serie de factores que modulan la forma en que el género se manifiesta en la participación en las comisiones u órganos de participación en la Universidad. Así, la tipología y composición del grupo, la experiencia profesional, la categoría profesional de los participantes, etc. 
influyen en la manera de tomar decisiones. La cantidad de personas que componen una reunión resulta una variable muy influyente en la calidad de la participación pudiendo decir que cuando el grupo es pequeño o mediano existe más participación, proporcionalmente hablando, que cuando el tamaño es muy grande. El estilo de liderazgo de la persona que coordina es otro factor que parece condicionar el tipo de intervención. Por ejemplo, en las reuniones coordinadas por mujeres parece que hay mayor participación de las mujeres ejerciendo el rol de co-iniciadoras y seguidoras. En general podríamos decir que cuando la reunión es dirigida por una mujer hay más participación y ésta es más homogénea (entre hombres y mujeres, de todos los roles y todos los scripts,...). Por lo tanto podríamos decir que la dirección de reuniones por parte de las mujeres aumentaría la calidad de la toma de decisiones si entendemos que ésta se ve beneficiada por la mayor y mejor distribución de la participación. Además del género de quien coordina una reunión parece que influye decisivamente en la calidad de la participación y de la toma de decisiones, el estilo de coordinación y/o tipo de liderazgo de quién dirige la reunión. A grandes rasgos, y con la debida cautela, es posible caracterizar un «estilo femenino» de participación en la línea que señalan Eagly y Johannsen-Schmidt. Éste vendría definido por el uso de intervenciones directas y concisas, la búsqueda del diálogo y el consenso, la articulación de planteamientos más pragmáticos, y la preocupación por los aspectos relacionados con el proceso y no sólo con el contenido de la toma de decisiones y esto afecta positivamente a la participación.

La calidad en la toma de decisiones depende tanto del grado en que la decisión adoptada contribuye a la consecución de los objetivos del grupo, como del proceso mismo de participación a través de cómo se toma la decisión. Entre los factores procesuales, revisten especial importancia la representación de los diferentes intereses implicados y la participación en términos de igualdad.

La dirección de grandes equipos de investigación sigue siendo patrimonio del género masculino mientras que no lo es la tarea investigadora. El liderazgo de grupos grandes de investigación lleva asociado una serie de funciones que no resultan atractivas para las investigadoras como por ejemplo la organización de grandes congresos que proporcionan gran visibilidad lo que no resulta de especial interés para ellas que sin embargo aprecian más satisfacción en lo intrínseco (Poole y Langan-Fox).

La existencia de cierta invisibilidad de las mujeres en las universidades estudiadas aparece como consecuencia de las características socioculturales que han impregnado la vida de las académicas universitarias así como de las circunstancias estructurales imperantes en la Universidad española (Sánchez, Tomàs y Lavié). 


\section{AGRADECIMIENTOS}

Este artículo no hubiera sido posible sin la colaboración de mis colegas en las diversas investigaciones de las que es fruto a quien quiero agradecer su inestimable implicación además de las personas informantes que las han hecho posible.

\section{REFERENCIAS BIBLIOGRÁFICAS}

Amancio, Lígia. «Reflections on science as a gendered Endeavour: changes and continuities». Social Science Information 44.1 (2005): 65-83.

Asmar, Christine. "Is there a gendered agenda in academia? The research experience of female and male $\mathrm{PhD}$ graduates in Australian universities». Higher Education 38.3 (1999): 255-273.

Bagilhole, Barbara. «Challenging women in the male academy: think about draining the swamp». Challenges and negotiations for women in higher education. Eds. Pamela Cotterill, Sue Jackson y GayleLetherby. Dordrecht: Springer, 2007, 21-32.

Bagilhole, Barbaray Kate White. «Created in their image: An analysis of male cultural hegemony in higher education in Australian and the United Kingdom». Re-searching research agendas: women, research and publication in higher education. In B. Groombridgey V. Mackie (eds.), Proceedings of the Australian Technology network-women's executive development (ATN-WEXDEV) 2003 Research Conference (1-12). Perth: Curtin University of Technology Learning Support Network. (2003).

Balwell, James W. y Joseph Berger. «Gender, status, and behavior in task situations».Social Psychology Quarterly 59.3 (1996): 273-283.

Britton, Carolyn. «Supporting women in research». Women, research and careers. Eds. Sue Hatt, Julie Kent y Carolyn Britton. Houndsmills: Macmillan (1999): 69-88.

CaceresReche, María Pilar, Antonio Sachicola y María Angustias Hinojo Lucena. «Análisis del liderazgo femenino y poder académico en el contexto universitario español.» European Scientific Journal ESJ 11 (2015): 296-313.

Carli, Linda L. «Gender and social influence». Journal of Social Issues 57 (2001): 725-741.

Dean, Elizabeth, Lesley Johnson, Gar Jones y Nicola Lengkeek. Women, research and research productivity in the post-1987 universities: Opportunities and constraints. University of Western Sydney, Nepean: Department of Employment, Education, Training and Youth Affairs, Evaluations and Investigations program, Higher education Division, (1996).

Dever, Marianne y Zoë Morrison. «Women, Research Performance and work Context». Tertiary Education and Management 15.1 (2009): 49-62. 
Doherty, Liz y Simonetta Manfredi. «Women's Progression to Senior Positions in English Universities». Employee Relations 28.6 (2006): 553-572.

Eagly, Alice H. y Mary C. Johannesen-Schmidt «The leadership styles of women and men», Journal of Social Issues 57.4 (2001): 781-797.

Forster, Nick. "A case study of women academics'views on equal opportunities, career prospects and work-family conflicts in a British university». Women in Management Review 15.7 (2000): 316-330.

Guillamón, Cristina y Marina Tomàs-Folch. El porqué del bajo porcentaje de mujeres directoras en la enseñanza. Trabajo de Investigación. UAB. 2002.

Hawkins, Katherine y Christopher B. Power. «Gender differences in questions asked during small decision-making group discussions». Small Group Research 30.2 (1999): 235-256.

Helgesen, Sally. The Female Advantage: Women's Ways of Leadership. Kindle Edition, 1990.

Kyvik, Svein, y Mari Teigen. «Child care, research collaboration, and gender differences in scientific productivity». Science, Technology \& Human Values 21.1, (1996): 54-71.

Kray, Laura J., Adam D. Galinsky y Leigh Thompson.»Reversing the gender gap in negotiations: an exploration of stereotype regeneration». Organizational Behavior and Human Decision Processes 87.2 (2002): 386-409.

Lafferty, George y Jenny Fleming. «The restructuring of academic work in Australia: Power, management and gender». British Journal of Sociology of Education 21.2 (2000): 257-267.

LePine, Jeffrey A., John R. Hollenbeck, Daniel R. Ilgen, Jason A. Colquitt y Aleksander Ellis. «Gender composition, situational strength, and team decision-making accuracy: a criterion decomposition approach». Organizational Behavior and Human Decision Processes 88.1 (2002): 445-475.

Mayer, Michael E. «Behaviors leading to more effective decisions in small groups embedded in organizations». Communication Reports 11.2 (1998): 123-132.

MEC (Unidad de Mujeres y Ciencia). Académicas en cifras 2007. 2007. 23 de abril del 2017.

Miqueo, Consuelo, María José Barral Morán y Carmen Magallón. Estudios iberoamericanos de género en ciencia, tecnología y salud: GENCIBER. Prensas Universitarias de Zaragoza. 2008.

Nájera, Elena. «¿Feminismo de la igualdad y feminismo de la diferencia?» Feminismo/s 15 (2010): 9-14

Olssen, Mark y Michael Peters. «Neoliberalism, higher education and the knowledge economy. From the free market to knowledge capitalism». Journal of Education Policy 20.3 (2005): 313-345.

Park, Shelley M. «Research, Teaching and service. Why shouldn't women's work count?». Journal of HigherEducation 67.1 (1996): 46-84. 
Pérez Sedeño, Eulalia. Las mujeres en el Sistema de Ciencia y Tecnología.» Estudio de casos. Madrid: Organización de Estados Iberoamericanos (OEI) (serie Cuadernos de Iberoamérica), 2001.

Pérez Sedeño, Eulalia. «Sociedad, Cultura y Tecnologías Reproductivas». Actas del IV Congreso de la Sociedad de Lógica, Metodología y Filosofía de la Ciencia en España. Valladolid: Sociedad de Lógica, Metodología y Filosofía de la Ciencia en España. 2004: 438-441.

Pérez Sedeño, Eulalia. Los programas de formación y movilidad del personal investigador de flujo directo e inverso: Problemas, retos y soluciones. Informe del Proyecto EA, 2005.

Phillips, Anne. Engendering democracy. University Park: Pennsylvania State University Press, 1991.

Poole, Millicent y Janice Langan-Fox. Australian women and careers: Psychological and contextual influences over the life course. Cambridge: Cambridge University Press, 1997.

Probert, Belinda. «Gender and unequal outcomes in academic careers». Gender, Work and Organisations 12.1 (2005): 50-72.

Ridgeway, Cecilia L. y Lynn Smith-Lovin. «The gender system and interaction». Annual Review of Sociology 25 (1999): 191-216.

Rutherford, Sarah. «Organizational cultures, women managers and exclusion». Women in Management Review 16.8 (2001): 371-382.

Sagaria, Mary y Lyndsay Agans. «Gender equality in US higher education: International framing and institutional realities». In Keiko Yokoyama (Ed.), Gender and higher education: Australia, Japan, the UK and USA. Ed. KeikoYokoyama. Hiroshima: Higher Education Institute Press, (2006): 47-68.

Sánchez-Moreno, Marita, Marina Tomás-Folch y José Manuel Lavié Martínez. «Visibilidad y poder de las mujeres en instituciones universitarias». Archivos Analíticos de Políticas Educativas 21.32 (2013).

Saunderson, Wendy. «Women, Academia and Identity: Constructions of Equal Opportunities in the 'New Managerialism'- A Case of 'Lipstick on the Gorilla'? » Higher Education Quarterly 56.4, ( 2002): 376-406.

Shackelford, Susan, Wendy Wood y Sthephen Worchel. «Behavioral styles and the influence of women in mixed-sex groups». Social Psychology Quarterly 59.3, (1996): 284-293.

Smeby, Jens-Christian y Suerre Try. «Departmental contexts and faculty research activity in Norway». Research in Higher Education 46.6 (2005): 593-619.

Smith-Lovin, Lynn y Charles Brody. «Interruptions in group discussions: the effects of gender and group composition». American Sociological Review, 54.3 (1989): 424-435.

Soliman, Izabel y Hani Soliman. Academic workload and quality. Assessment and Evaluation in Higher Education, 22.2 (1997), 135-157. 
Toren, Nina. «The temporal dimension of gender inequality in academic». Higher Education, 25.4 (1993): 439-455.

Webster, Berenika M. «Polish women in science: A bibliometric analysis of Polish science and its publications, 1980-1999». Research Evaluation 10.3, (2001):185-194.

Vendrell, Esteve. Dinámica de Grups i Psicologia dels Grups. Barcelona: UB, 1999. 